\title{
RETRACTED ARTICLE: Isolation and Functional Characterization of an Antifreeze Protein Gene, TaAFPIII, from Wheat (Triticum aestivum)
}

Libao Cheng $\cdot$ Xuan Gao $\cdot$ Hussain Javeed $\cdot$ Li Zhang Jiutong Sun • Yanzhen He $\cdot$ Hong Zhang •

Zhijuan Feng • Guangxiao Yang • Guangyuan He

Published online: 28 February 2012

(C) Springer-Verlag 2012

This article has been retracted due to fraudulent content with serious consequences to wheat production and its economic impact.

This article has been retracted due to fraudulent content with serious consequences to wheat production and its economic impact.

L. Cheng $\cdot$ X. Gao $\cdot$ H. Javeed $\cdot$ L. Zhang $\cdot$ J. Sun $\cdot$ Y. He $\cdot$

H. Zhang $\cdot$ Z. Feng $\cdot$ G. Yang $\cdot$ G. He $(\triangle)$

China-UK HUST-RRes Genetic Engineering and Genomics Joint Laboratory, Genetic Engineering International Cooperation Base of MoST, Key Laboratory of Molecular Biophysics MoE,

College of Life Science and Technology,

Huazhong University of Science and Technology,

Wuhan 430074, People's Republic of China

e-mail: hegy@hust.edu.cn

L. Cheng

School of Horticulture and Plant Protection, Yangzhou University,

Jiangsu, People's Republic of China 